\title{
Nonvanishing of algebraic entropy for geometrically finite groups of isometries of Hadamard manifolds *
}

\author{
Roger C. Alperin \\ San Jose State University, San Jose, CA, USA \\ Gennady A. Noskov \\ Bielefeld University, GERMANY and \\ Institute of Mathematics, Omsk, RUSSIA
}

February 14, 2003

\begin{abstract}
We prove that any nonelementary geometrically finite group of isometries of a pinched Hadamard manifold has nonzero algebraic entropy in the sense of M. Gromov. In other words it has a uniform exponential growth,
\end{abstract}

\section{Introduction}

Given a group $\Gamma$ generated by a finite set $S$ we denote by $B_{S}(r)$ the the ball of radius $k$ in the Cayley graph of $\Gamma$ relative to $S$. The exponential growth rate or $\omega(\Gamma, S)=\lim _{k \rightarrow \infty} k \sqrt{\left|B_{S}(k)\right|}$ is well defined (by submultiplicativity). We set

$$
\omega(\Gamma)=\inf \{\omega(\Gamma, S) \mid S \text { is a finite generating set for } \Gamma\} .
$$

*Preprint is available online at the server of the Forchergruppe "Spektrale Analysis, asymptotische Verteilungen und stochastische Dynamik", Bielefeld University, http:// www.mathematik.uni-bielefeld.de /fgweb 
The group $\Gamma$ is of exponential growth if $\omega(\Gamma, S)>1$ for some (and hence for every) generating set $S$ and of uniform exponential growth if $\omega(\Gamma)>1$. (M. Gromov calls $\ln \omega$ the entropy of $\Gamma$ - the reason is that if $\Gamma$ is the fundamental group of a compact Riemannian manifold of unit diameter, then $\ln \omega(\Gamma, S)$ is a lower bound for the topological entropy of the geodesic flow of the manifold [Man81]). This notion should not be confused with the one given in [Ave72], where the notion of entropy is defined for the random walk on the group associated to the probability measure on the set of generators.

Clearly uniform exponential growth implies exponential growth. "On first sight, however, there seems to be no reason for the converse to be true" [Gro99]. Recently and rather spectacularly, J. S. Wilson has given example of exponential growth group which is not uniform [Wil02]; it is a group of automorphisms of a rooted tree.

The most common way to prove uniformity of exponential growth is to prove the UFS-property. We say that $\Gamma$ satisfies UFS-property (=uniformly contains free nonabelian semigroup) if there is a constant $n_{\Gamma} \geq 1$ such that for every generating set $A$ of $\Gamma$ there exist two elements in $\Gamma$ of word length $\leq n_{\Gamma}$, freely generating a free semigroup of rank 2 . The UFG-property, obtained from the previous one by changing free semigroup to free group is stronger.

Our main result is the following.

Theorem 1.1 Any nonelementary geometrically finite group of isometries of a pinched Hadamard manifold satisfies the UFG-property and hence has nonzero entropy.

We mention a few ideas about the proof of our main result given here. If $\Gamma$ is cocompact then it is word hyperbolic and the theorem follows from Koubi's result [Kou98] (which was known before due to M. Gromov [Gro87] and T. Delzant [Del96] in the torsionfree case.) In the main case of a noncocompact lattice we make use the geometry of neutered space associated to the group.

For a survey on this subject see the book of Gromov and the articles [GdlH97],[dlH02]. We mention now some related results. A group is called large if it has a subgroup of finite index which has a homomorphism onto a free non-abelian group. For example, in [GG00], largeness is proven for the noncocompact lattices in 3-dimensional hyperbolic space.

Also in our article [AN02], partial results were obtained for subgroups of $\mathrm{SL}_{2}$ in characteristic zero, but more complete results for non-zero characteristic. 
Theorem 1.2 If a finitely generated subgroup $\Gamma$ of $\mathrm{GL}_{2}(K), K$ a field of nonzero characteristic has an exponential growth then it satisfies the UFGproperty and consequently has uniform exponential growth.

A. Eskin, S. Mozes and H. Oh have recently given the 'uniform' proof of Tits' theorem in their beautiful result.

Theorem 1.3 [EMO01] Any finitely generated non-virtually solvable linear group over a field of characteristic zero has uniform exponential growth.

\section{Miscellany about $\delta$-hyperbolic spaces}

Let $X$ be a metric space. We denote by $|x-y|$ the distance between the points $x$ and $y$ in $X$. If $X$ is equipped with a basepoint $x_{0}$ then we shall use the notation $|x|=\left|x-x_{0}\right|, x \in X$. One of definitions of a hyperbolic space $X$ is given by means of the Gromov product relative to a base point $x_{0}$

$$
(x \cdot y)=(x \cdot y)_{x_{0}}=\frac{1}{2}(|x|+|y|-|x-y|), x, y \in X
$$

as follows: A metric space $X$ is called hyperbolic [Gro87] if there exists a constant $\delta \geq 0$ such that for every triple $x, y, z \in X$ and for every choice of a basepoint the following holds

$$
(x \cdot y) \geq \min ((x \cdot z),(y \cdot z))-\delta .
$$

A tripod $T$ is a union of three segments in $\mathbb{R}^{2}$, which have only the origin in common. Every geodesic triangle $\Delta=x y z$ in a geodesic metric space $X$ can be mapped onto a tripod so that the restriction of the map to each side of $\Delta$ is an isometry. We will call a map with these properties a tripod map. The tripod map always exists and is essentially unique. The three points $x^{\prime}, y^{\prime}, z^{\prime}$ on the sides opposite to $x, y, z$ respectively, whose image by the tripod map is equal to 0 describe an inscribed triangle and are called the internal vertices of $\Delta$. A geodesic triangle $\Delta$ is called $\delta$-thin for $\delta \geq 0$, if the fibers of the tripod map $f: \Delta \rightarrow T$ are of diameter at most $\delta$.

Lemma 2.1 Any geodesic triangle in a $\delta$-hyperbolic space $H$ is $4 \delta$-thin. 
Proof. See $\left[\mathrm{ABC}^{+}\right.$91] or [CDP90].

Let $x y z$ be a geodesic $\delta$-thin triangle with the inscribed triangle $x^{\prime} y^{\prime} z^{\prime}$. Let $\sigma_{y}(t), \sigma_{z}(t)$ be the arc length parameterizations of the segments $[x, y],[x, z]$ respectively such that at the moment $t=0$ they are located in $x$. We extend the parameterization for all $t \geq 0$ making the paths stop when they reach the vertices. Then by definition of thinness and lemma 2.1 the points $\sigma_{y}(t), \sigma_{z}(t)$ are distance at most $4 \delta$ apart until the moment when they reach the vertices $y^{\prime}, z^{\prime}$ of the inscribed triangle, that is

$$
\left|\sigma_{y}(t)-\sigma_{z}(t)\right| \leq 4 \delta, 0 \leq t \leq T=\frac{1}{2}(|x-y|+|x-z|-|y-z|)=(y \cdot z)_{x} .
$$

We say that $\sigma_{y}(t), \sigma_{z}(t) 4 \delta$-fellow travel each other on the segment $[0, T]$. More generally we have the following

Definition 2.2 The paths $\sigma(t), \tau(t) \varepsilon$-fellow travel each other on the segment $\left[t_{0}, t_{1}\right]$ if $|\sigma(t)-\tau(t)| \leq \varepsilon, t_{0} \leq t \leq t_{1}$. The function $\ell(\sigma(t), \tau(t), \varepsilon), \varepsilon>0$ is the supremum of the lengths of all time intervals on which $\sigma(t), \tau(t) \varepsilon$-fellow travel.

Lemma 2.3 (Fellow traveller property) Let xyz be a geodesic triangle in a $\delta$-hyperbolic space $X$ and $|y-z|=c>0$. Let $\sigma_{y}(t), \sigma_{z}(t)$ be the arc length parameterizations of the the segments $[x, y]$ and $[x, z]$. Then

1) $\left|\sigma_{y}(t)-\sigma_{z}(t)\right| \leq c+8 \delta$ for all $t \geq 0$.

2) For the reverse parameterizations $\sigma_{y}^{-}(t), \sigma_{z}^{-}(t)$ which start from $y, z$ respectively, we have $\left|\sigma_{y}^{-}(t)-\sigma_{z}^{-}(t)\right| \leq c+8 \delta, t \geq 0$.

3) Suppose that $\gamma, \gamma^{\prime}$ are geodesics with beginning and ending distance at most $c>0$. We have $\left|\gamma(t)-\gamma^{\prime}(t)\right| \leq 16 \delta+2 c$ for all $t \geq 0$.

Proof. 1) Consider the inscribed triangle for $x y z$. If both $\sigma_{y}(t)$ and $\sigma_{z}(t)$ do not reach the vertices of inscribed triangle then $\left|\sigma_{y}(t)-\sigma_{z}(t)\right| \leq 4 \delta$. If they do reach then there are points $y^{\prime}, z^{\prime}$ on the side $y, z$ distance at most $4 \delta$ from $\sigma_{y}(t), \sigma_{z}(t)$ respectively hence $\left|\sigma_{y}(t)-\sigma_{z}(t)\right| \leq c+8 \delta$.

2) If both $\sigma_{y}^{-}(t)$ and $\sigma_{z}^{-}(t)$ do not reach the vertices of inscribed triangle then arguing as in the previous case we obtain that $\left|\sigma_{y}^{-}(t)-\sigma_{z}^{-}(t)\right| \leq c+8 \delta$. In contrast to the previous case it is possible, that $\sigma_{y}^{-}(t)$ has reached the vertex of inscribed triangle but $\sigma_{z}^{-}(t)$ has not. If this happens, then let $z^{\prime}$ be a point on $[z, x]$ such that $\left|\sigma_{y}^{-}(t)-x\right|=\left|z^{\prime}-x\right|$. We have $\left|\sigma_{z}^{-}(t)-z^{\prime}\right|=|| z-x \mid-$ 

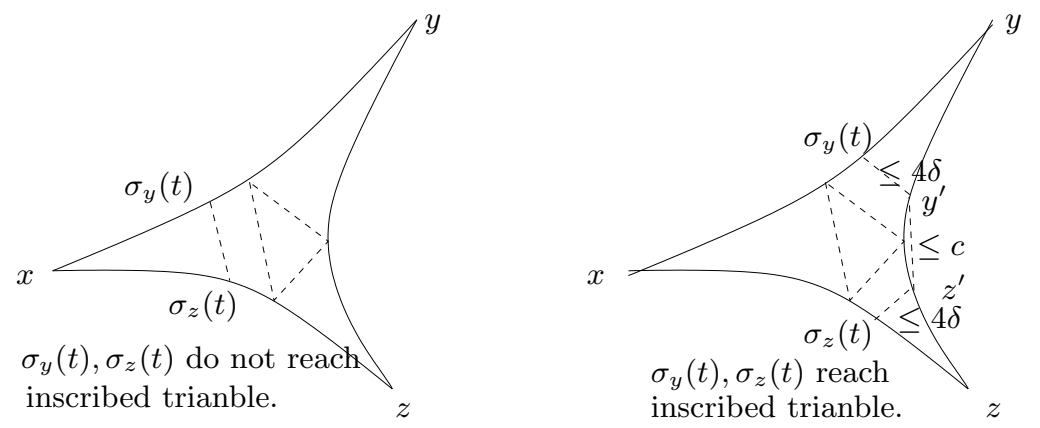

Figure 1: Fellow traveller property. Case 1).

$|y-x| \mid \leq c$, (see Figure 2). We conclude that $\left|\sigma_{y}^{-}(t)-\sigma_{z}^{-}(t)\right| \leq c+4 \delta, t \geq 0$. Finally if both $\sigma_{y}^{-}(t)$ and $\sigma_{z}^{-}(t)$ reach the vertices of inscribed triangle then again choosing suitable $z^{\prime}$ (or $y^{\prime}$ ) as above we get $\left|\sigma_{y}^{\prime}(t)-\sigma_{z}^{\prime}(t)\right| \leq c+4 \delta$.

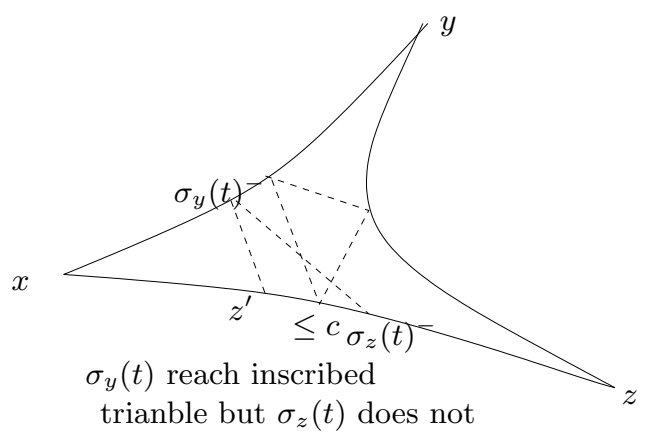

Figure 2: Fellow traveller property. Case 2)

3) Easily follows applying 1) and 2) to the triangles $\gamma(0) \gamma(\infty) \gamma^{\prime}(\infty)$ and $\gamma^{\prime}(0) \gamma(\infty) \gamma^{\prime}(\infty)$ respectively.

We say that the geodesic triangle $x y z$ has an obtuse $y$-angle if either $y$ is the nearest to $x$ point on the side $[y, z]$ or $y$ is the nearest to $z$ point on the side $[y, x]$.

Lemma 2.4 (Obtuse-angled triangle lies in $8 \delta$-neighbourhood of the side) Let $x y z$ be a geodesic triangle with obtuse $y$-angle, then $|x-z| \geq|x-y|+\mid y-$ $z \mid-16 \delta$. Furthermore, $(x \cdot z)_{y} \leq 8 \delta$.

Proof. Use the inscribed triangle. Say $y$ is nearest to $x$ on the side $[y, z]$. Since $\left|z^{\prime}-x^{\prime}\right| \leq 4 \delta$ we conclude that $\left|z^{\prime}-y\right| \leq 4 \delta$ hence $\left|y-y^{\prime}\right| \leq 8 \delta$. 
Thus $|x-z|=\left|x-y^{\prime}\right|+\left|y^{\prime}-z\right| \geq|x-y|-\left|y-y^{\prime}\right|+|y-z|-\mid y-$ $y^{\prime}|\geq| x-y|+| y-z \mid-16 \delta$. Finally, applying the last inequality we obtain $(x \cdot z)_{y}=\frac{1}{2}(|x-y|+|z-y|-|z-x|) \leq 8 \delta$.

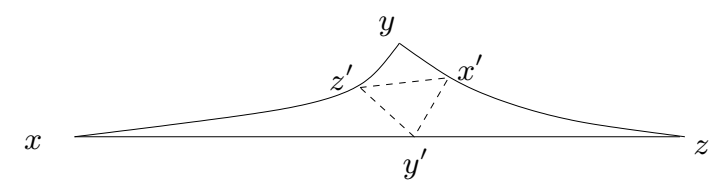

Figure 3: Obtuse triangle inequality.

The next lemmas help us figure out the structure of geodesic polygons;

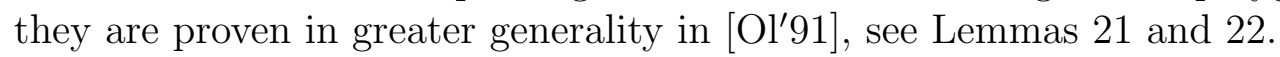

Lemma 2.51 1) Suppose that a geodesic 4-gon $\left[x_{1}, x_{2}, x_{3}, x_{4}\right]$ satisfies the following conditions: $\left|x_{i+1}-x_{i}\right|>180 \delta$ for $i=1,2,3$ and $\left(x_{1} \cdot x_{3}\right)_{x_{2}},\left(x_{2} \cdot x_{4}\right)_{x_{3}} \leq$ $14 \delta$ for. Then the polygonal line $p=x_{1} x_{2} x_{3} x_{4}$ is contained in the closed $28 \delta$ neighborhood of the side $\left[x_{1}, x_{4}\right]$. In particular, $\left|x_{1}-x_{4}\right|>\sum_{1}^{3}\left|x_{i+1}-x_{i}\right|-$ $168 \delta$.

2) Suppose that a geodesic 5-gon $\left[x_{1}, x_{2}, x_{3}, x_{4}, x_{5}\right]$ satisfies the following conditions: $\left|x_{i+1}-x_{i}\right|>180 \delta$ for $i=1,2,3,4$ and $\left(x_{i+2} \cdot x_{i}\right)_{x_{i+1}} \leq 14 \delta$ for $i=1,2,3$. Then the polygonal line $p=x_{1} x_{2} x_{3} x_{4} x_{5}$ is contained in

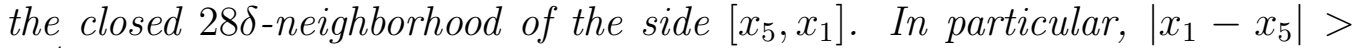
$\sum_{1}^{4}\left|x_{i+1}-x_{i}\right|-168 \delta$.

We have the following variant of the above lemma for "small" sides $\left[x_{2}, x_{3}\right],\left[x_{4}, x_{5}\right]$.

Lemma 2.6 Suppose that a geodesic 5-gon $\left[x_{1}, x_{2}, x_{3}, x_{4}, x_{5}\right]$ satisfies the conditions $\left|x_{2}-x_{3}\right|,\left|x_{4}-x_{5}\right| \leq 180 \delta$. Then

$$
\left|x_{1}-x_{5}\right|>\left|x_{1}-x_{2}\right|+\left|x_{3}-x_{4}\right|-360 \delta-2 \ell_{0},
$$

where $\ell_{0}=\ell\left(\gamma, \gamma^{\prime}, 380 \delta\right)$ - the time of $380 \delta$-fellow travel of geodesics $\gamma=$ $\left[x_{2}, x_{1}\right], \gamma^{\prime}=\left[x_{3}, x_{4}\right]$.

Proof. First note that $\ell_{0} \geq\left(x_{1} \cdot x_{5}\right)_{x_{2}}$. Indeed, the geodesics along the segments $\left[x_{2}, x_{1}\right],\left[x_{2}, x_{5}\right] \quad 4 \delta$-fellow travel each other until the moment $t=\left(x_{1} \cdot x_{5}\right)_{x_{2}}$ by the thinness property, see inequality (3). Geodesics 
$\left[x_{2}, x_{5}\right],\left[x_{3}, x_{4}\right] \quad 376 \delta$-fellow travel each other all the time by the assertion 3 ) of Lemma 2.3. Thus the segments $\left[x_{2}, x_{1}\right],\left[x_{3}, x_{4}\right] \quad 380 \delta$-fellow travel each other until the moment $t=\left(x_{1} \cdot x_{5}\right)_{x_{2}}$, hence $\ell_{0} \geq\left(x_{1} \cdot x_{5}\right)_{x_{2}}$. Finally, $\left|x_{1}-x_{5}\right|=\left|x_{1}-x_{2}\right|+\left|x_{2}-x_{5}\right|-2\left(x_{1} \cdot x_{5}\right)_{x_{2}} \geq\left|x_{1}-x_{2}\right|+\left|x_{3}-x_{4}\right|-360 \delta-$ $2\left(x_{1} \cdot x_{5}\right)_{x_{2}}>\left|x_{1}-x_{2}\right|+\left|x_{3}-x_{4}\right|-360 \delta-2 \ell_{0}$.

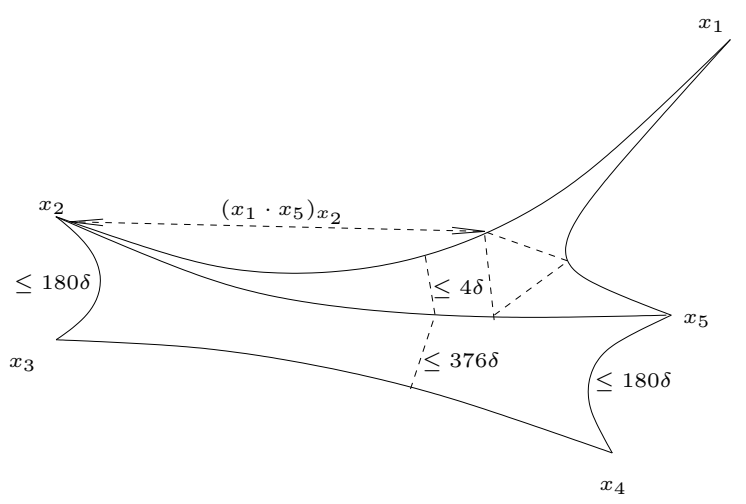

Figure 4: Lemma 2.6: Divergence for 5-gons with two small sides.

\section{Uniformly hyperbolic isometries in groups acting on $\delta$-hyperbolic spaces}

An isometry $g$ of a $\delta$-hyperbolic space $X$ is hyperbolic if there is a point $x \in$ $X$ such that the map $n \mapsto g^{n} x$ from $\mathbb{Z}$ to $X$ is a quasi-isometric embedding, [CDP90]. This means that $\left|g^{n}(x)-x\right| \geq c n$ for a suitable positive constant $c$ and every natural $n$. The axis of $g$ is a bi-infinite geodesic on which $g$ acts as a translation. In general, a hyperbolic isometry $g$ does not need to possess an axis, however it does in case $X$ is a pinched Hadamard manifold (they are complete CAT(0)-spaces). Or $X$ is a Cayley graph of a word hyperbolic group $\Gamma$ and $g=h^{n}$ for a constant $n=n(\Gamma)$ and an element $h$ is of infinite order [Del96].

Lemma 3.1 ([CDP90], lemma 9.2.2.) Suppose that for an isometry $g$ of a $\delta$-hyperbolic space $X$ there is a point $x \in X$ such that

$$
\left|g^{2} x-x\right|>|g x-x|+2 \delta \text {. }
$$

Then $g$ is hyperbolic. 
Lemma 3.2 ([CDP90], lemma 9.2.3.) Suppose that $\delta>0$. Let $g, h \in I$ som $X$ be such that for some $x \in X$ the following holds

$$
\min \{|g x-x|,|h x-x|\} \geq 2(g x \cdot h x)_{x}+6 \delta .
$$

Then $g h, h g$ are hyperbolic.

Let $S$ be a finite set of isometries of a $\delta$-hyperbolic space $X$. The size of $S$ at $x \in X$ is

$$
|S|_{x}=\max _{s \in S}|s x-x| .
$$

The following is a variant of Proposition 3.2 from [Kou98] for actions of a hyperbolic group.

Lemma 3.3 (Long generating system gives rise to a short hyperbolic isometry) Let $X$ be a geodesic $\delta$-hyperbolic space and let $\Gamma$ be a group of isometries of $X$, generated by a finite set of isometries $S$. Suppose that $|S|_{x}>100 \delta$ for each $x \in X$. Then there is a hyperbolic isometry in $\Gamma$ which is a product of at most 2 isometries from $S$.

Proof. ([Kou98]) Suppose that $|S|_{x}>100 \delta$ for each $x \in X$ and that $S$ consists of nonhyperbolic isometries. Let $x_{0}$ be a point of almost minimal displacement for $S$ that is

$$
|S|_{x_{0}} \leq \inf _{y \in X}|S|_{y}+\delta .
$$

Let $S_{0}=\left\{a \in S:\left|a x_{0}-x_{0}\right| \geq 50 \delta\right\}$ be the long part of $S$. Fix $a_{1} \in S$ such that $\left|a_{1} x_{0}-x_{0}\right|=\max _{a \in S}|a x-x|>100 \delta$.

Case 1): There is $a \in S_{0}$ such that $\left(a_{1} x_{0} \cdot a x_{0}\right)_{x_{0}} \leq 20 \delta$. Applying the lemma 3.2 we obtain that $a a_{1}$ is hyperbolic.

Case 2): $\left(a_{1} x_{0} \cdot a x_{0}\right)_{x_{0}} \geq 20 \delta$ for all $a \in S_{0}$. We will show that this assumption leads to a contradiction of the almost minimality of $x_{0}$. Namely, we shall show that for the point $x_{1}$ on the geodesic $\left[x_{0}, a_{1} x_{0}\right]$ such that $\mid x_{1}-$ $x_{0} \mid=20 \delta$, the following holds

$$
|S|_{x_{1}}<|S|_{x_{0}}-10 \delta
$$

and this contradicts the almost minimality of $x_{0}$.

Choose $x_{a} \in\left[x_{0}, a x_{0}\right]$ so that $\left|x_{a}-x_{0}\right|=20 \delta$, then, since $\left(a_{1} x_{0} \cdot a x_{0}\right)_{x_{0}} \geq$ $20 \delta$, by applying the inequality (3) to the triple of points $\left(x_{0}, a_{1} x_{0}, a x_{0}\right)$, we have that the $\left|x_{1}-x_{a}\right| \leq 4 \delta$. Consider $x_{1}$ as a new base point and let $a \in S$.

We assert the following: 


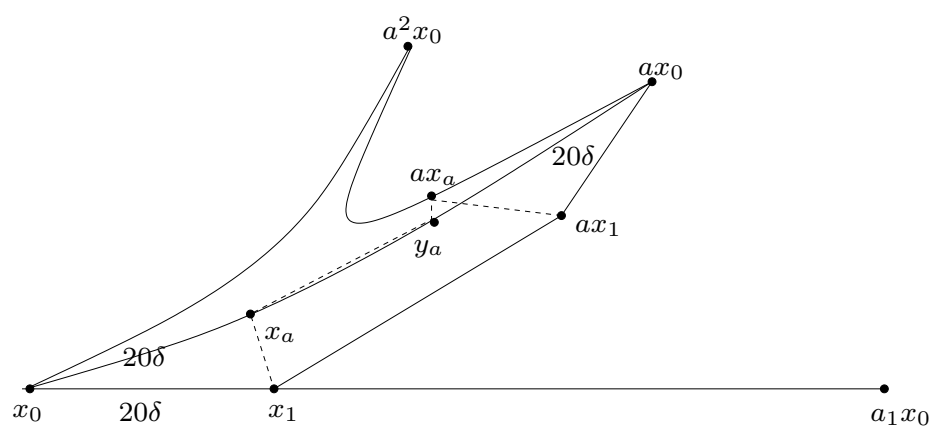

Figure 5: Long generating system gives rise to a uniform hyperbolic isometry.

- if $a \in S_{0}$, then $\left|a x_{1}-x_{1}\right| \leq\left|a x_{0}-x_{0}\right|-28 \delta$;

- if $a \notin S_{0}$, then $\left|a x_{0}-x_{0}\right| \leq 90 \delta$.

It follows from this assertion that

$$
\max _{a \in S}\left|a x_{1}-x_{1}\right| \leq \max \left\{\max _{a \in S}\left|a x_{0}-x_{0}\right|-28 \delta, 90 \delta\right\}<\max _{a \in S}\left|a x_{0}-x_{0}\right|-10 \delta,
$$

contradicting the almost minimality of $x_{0}$.

To prove the assertion, consider as a first case when $a \in S_{0}$. Choose $y_{a} \in\left[x_{0}, a x_{0}\right]$ so that $\left|y_{a}-a x_{0}\right|=20 \delta$. We have

$\left(a^{2} x_{0} \cdot x_{0}\right)_{a x_{0}}=\frac{1}{2}\left(\left|a x_{0}-x_{0}\right|+\left|a^{2} x_{0}-a x_{0}\right|-\left|a^{2} x_{0}-x_{0}\right|\right) \geq \frac{1}{2}\left|a x_{0}-x_{0}\right|-\delta \geq 24 \delta$.

Applying inequality (3) to the triangle $\Delta\left(x_{0}, a x_{0}, a^{2} x_{0}\right)$ we obtain $\left|a x_{a}-y_{a}\right| \leq$ $4 \delta$. By assumption $\left(a_{1} x_{0} \cdot a x_{0}\right)_{x_{0}} \geq 20 \delta$ hence $\left|a x_{a}-a x_{1}\right|=\left|x_{a}-x_{1}\right| \leq 4 \delta$.

We conclude that

$$
\begin{aligned}
\left|a x_{1}-x_{1}\right| \leq & \left|a x_{1}-a x_{a}\right|+\left|a x_{a}-y_{a}\right|+\left|y_{a}-x_{a}\right|+\left|x_{a}-x_{1}\right| \leq \\
& 4 \delta+4 \delta+\left(\left|a x_{0}-x_{0}\right|-40 \delta\right)+4 \delta \\
\leq & \left|a x_{0}-x_{0}\right|-28 \delta .
\end{aligned}
$$

For the second case when $a \notin S_{0}$ we have

$$
\begin{aligned}
\left|a x_{1}-x_{1}\right| & \leq\left|a x_{1}-a x_{0}\right|+\left|a x_{0}-x_{0}\right|+\left|x_{0}-x_{1}\right| \leq \\
20 \delta+50 \delta+20 \delta & \leq 90 \delta
\end{aligned}
$$




\section{Geometrically finite groups of isometries of Hadamard manifolds}

An Hadamard manifold is a simply connected, complete Riemannian manifold without boundary and with nonpositive sectional curvatures. We will assume moreover that $X$ is pinched, i.e. all the sectional curvatures lie between two negative constants: $-\kappa^{2} \leq K(X) \leq-1$, [Bow95]. A convenient reference for such manifolds is [BGS85].

A basic fact about Hadamard manifold is that the exponential map based at any point is injective. Thus, any such manifold $X$ is diffeomorphic to $\mathbb{R}^{n}$. $X$ can be naturally compactified by adjoining an ideal sphere $\partial X$ to $X$, so that $\bar{X}=X \cup \partial X$ is homeomorphic to a closed $n$-dimensional ball. A pinched Hadamard manifold is a visibility manifold, i.e. any two points $x, y \in \bar{X}$ can be joined by a unique geodesic, which we denote by $[x, y]$. When we speak of geodesic as paths, it will be assumed they are parameterized by arc-length. Denote by $|x-y|$ a Riemannian path distance between $x, y \in X$. The point $\xi \in \partial X$ can be represented by a geodesic ray $c_{\xi}:[0, \infty) \rightarrow X$. The function $h_{c}(x)=\lim _{t \rightarrow \infty}(|x-c(t)|-t)$ is called the horofunction about $\xi$ associated to the geodesic ray $c$. It turns out that $h$ is $C^{2}$ [HIH77] and the norm of its gradient is everywhere equal to 1 . The level sets of $h_{c}$ are called horospheres about $\xi$. The horospheres form a codimension 1 foliation of $X$ orthogonal to the foliation by bi-infinite geodesics having one endpoint at $\xi$. A set of the form $h_{\xi}^{-1}[r, \infty)$ for $r \in \mathbb{R}$ is called a horoball about $\xi$. Such a horoball may alternatively be described as the closure of the set $\cup\left\{\left\{B_{t}(\beta(t)): t \in(0, \infty)\right\}\right.$ where $\beta$ is a geodesic ray tending to $\xi$ with $\beta(0) \in h_{\xi}^{-1}(r)$. In particular horoballs are convex.

The well known classification of isometries of a hyperbolic space holds for $X$ too. Any isometry $g$ of $X$ extends to a homeomorphism of $\bar{X}$. We shall write $\bar{X}^{g}$ for the set of fixed points of $g$ in $\bar{X}$. Any nonidentical isometry $g$ of $X$ is precisely on of the following types:

1) $g$ is elliptic, that is $X^{g}$ is nonempty,

2) $g$ is parabolic, that is $\bar{X}^{g}$ consists of a single point $p \in \partial X$ and $g$ preserves setwise each horosphere about $p$,

3) $g$ is hyperbolic, that is $\bar{X}^{g}=\{p, q\}$, where $p$ and $q$ are distinct points of $\partial X$. In this case $g$ translates along the axis - the geodesic connecting $p$ and $q$ and we denote by $\|g\|$ the amplitude of this translation (= translation length. 
Note that this definition of hyperbolicity is consistent with the one given before for isometries of a $\delta$ - hyperbolic space.

By definition, the group $\Gamma \leq$ Isom $X$ is elementary if $\bar{X}^{\Gamma} \neq \emptyset$ or else $\Gamma$ preserves bi-infinite geodesic in $\bar{X}$.

Definition 4.1 Let $X$ be a pinched Hadamard manifold and $\Gamma \leq \operatorname{Isom} X$ is a subgroup of isometry group, acting properly. $\Gamma$ is geometrically finite if the following properties hold:

- there is a $\Gamma$-invariant family (possibly empty) $\mathcal{B}=\left\{B_{p} \mid p \in P\right\}$ of pairwise disjoint closed horoballs in $X$ with $\Gamma \backslash \mathcal{B}$ finite;

- there is a non-empty closed convex $\Gamma$-invariant subset $Z \subseteq X$ such that the $\Gamma$-action on the neutered space $X_{\Gamma}=Z-\bigcup_{p \in P} \operatorname{Int}\left(B_{p}\right)$ is cocompact.

The more restrictive class of groups, consisting of lattices, corresponds to the case of $Z=X$ in the above definition.

We now return to the discussion of the geometry of $X_{\Gamma}$. Each maximal parabolic subgroup $P$ of $\Gamma$ fixes a point at infinity of $X$ and hence fixes any horoball in $X$ centered at this point. We consider $X_{\Gamma}=Z-\bigcup_{P \in \mathbb{P}} \operatorname{Int}\left(B_{P}\right)$ with the path metric, that is the metric given by lengths of paths, computed using the standard hyperbolic Riemannian metric. $X_{\Gamma}$ is complete and locally compact, so it is a geodesic metric space. $\Gamma$ acts on $X$ by isometries, with finite stabilizers, and with compact quotient.

We call the boundary piece $S_{P}=X \cap \partial B_{P}$ that results from removing $\operatorname{int}\left(B_{P}\right)$ a horosphere of $X$. Two horospheres $S_{P}$ and $S_{P^{\prime}}$ are have the same image in $M$ if and only if $P$ and $P^{\prime}$ are conjugate in $\Gamma$.

Since $Z$ is convex, the metric on $Z$ is the restriction of the metric on $X$. In particular, it is a geodesic metric space, geodesics are unique, and they vary continuously with choice of endpoint. For points $x, y \in X$ we will denote $d_{X}(x, y)$ their distance apart in $X$ and $d_{X_{\Gamma}}(x, y)$ their path distance in $X_{\Gamma}$. The path metric on $X_{\Gamma}$ is at most exponentially distorted with respect to $d_{X}$; namely

$$
d_{X}(x, y) \leq d_{X_{\Gamma}}(x, y) \leq \sinh \left(\kappa^{2} \cdot d_{X}(x, y)\right)
$$

for all $x, y \in X$. This is proven in [Far95] for the case of lattices but the proof goes equally well for geometrically finite groups. Thus, there is a monotonically increasing positive function $\phi$, does not depending on $\Gamma$ such that $\phi(x) \rightarrow \infty$ with $x \rightarrow \infty$ and such that $d_{X}>\phi\left(d_{X_{\Gamma}}\right)$.

By a result of Bowditch all $P$ are virtually nilpotent [Bow95] (this follows from the Margulis Lemma). 


\section{Uniform hyperbolic elements in groups act- ing on Hadamard manifolds}

In the case of noncocompact discrete actions we know of no results about uniform hyperbolic elements as proven in the previous section. But we have

Lemma 5.1 Let $\Gamma$ be a nonelementary geometrically finite group acting on a Hadamard manifold $X$. There is neutered space $X_{\Gamma}$ and a constant $\Delta>0$ such that if $\Gamma$ is generated by the set $S$ and $|S|_{x}^{X_{\Gamma}}>\Delta$ for each $x \in X$, then there is an hyperbolic isometry in $\Gamma$ which is a product of at most 2 isometries from $S$. (The norm is taken relative to the path metric on $X_{\Gamma}$.)

Proof. Take any $\Delta>0$ such that $\phi(\Delta)>100 \delta$, where $\phi$ is defined in the previous section. Choose the horoball system so that that for any two of them $\phi\left(d_{X_{\Gamma}}\left(B_{1}, B_{2}\right)\right)>100 \delta$. Now take the generating set $S$ so that $|S|_{x}^{X_{\Gamma}}>\Delta$ for each $x \in X_{\Gamma}$. Then $|S|_{x}^{X}>\phi(\Delta)>100 \delta, x \in X_{\Gamma}$. To apply the Lemma 3.3 above it is enough to ensure that $|S|_{x}^{X}>100 \delta$ for all $x \in X$. We have already done this for $x \in X$. Now suppose $x$ belongs to some horoball $B$. If $S x \subset B$, then $\Gamma$ preserves the horoball $B$ and hence is elementary - contradiction. Thus $s x \notin B$ for some $s \in S$. The segment $[x, s x]$ crosses the horospheres $\partial B, s \partial B$ in some points $x_{1}, x_{2}$ and we have $|s x-x|_{X} \geq\left|x_{2}-x_{1}\right|_{X} \geq \phi\left(\left|x_{2}-x_{1}\right|_{X_{\Gamma}}\right) \geq \phi\left(d_{X_{\Gamma}}(B, s B)\right)>100 \delta$. Thus, we can apply the Lemma 3.3.

Theorem 5.2 A geometrically finite group $\Gamma$, acting on a pinched Hadamard manifold $X$, uniformly contains hyperbolic elements.

Proof. Let $X$ be a corresponding neutered space, obtained by removing from the convex hull of the limit set the disjoint $\Gamma$-invariant union of horoballs about parabolic fixed points. By Lemma 5.1 there is a $\Delta>0$ such that if $\Gamma$ is generated by $S$ and $|S|_{x}^{X_{\Gamma}}>\Delta$ for each $x \in X_{\Gamma}$, then there is a hyperbolic isometry in $\Gamma$ which is a product of at most 2 isometries from $S$. (The norm is taken relative to the path metric on $X_{\Gamma}$.) Denote by $\mathcal{G}_{0}$ the set of all finite generating sets $S$ for $\Gamma$ such that $|S|_{x}^{X_{\Gamma}} \leq \Delta$ for some $x \in X_{\Gamma}$. Since the action on $X_{\Gamma}$ is proper and cocompact, the set $\mathcal{G}_{0}$ is finite up to conjugacy. Denote by $\mathcal{G}_{1}$ the set of all finite generating sets $S$ for $\Gamma$ do not belonging to $\mathcal{G}_{0}$. We want to get a universal bound for the length of a shortest hyperbolic element in an arbitrary generating system. Firstly it is easy to do this for 
$\mathcal{G}_{0}$. Indeed, this length is invariant under conjugation, and $\mathcal{G}_{0}$ is finite up to conjugacy. Next, if $|S|_{x}^{X_{\Gamma}}>\Delta$ for each $x \in X$, then by the proposition above there is a hyperbolic isometry in $\Gamma$ which is a product of not more than 2 isometries from $S$.

\section{Separation of axes}

Definition 6.1 By an axis of an isometry $g$ of a metric space $X$ we call in isometric copy of $\mathbb{R}$ inside $X$ on which $g$ acts by translation. We say that the group $\Gamma$ of isometries of a metric space $X$ satisfies $S A$-property (= Separation of axes) if for every $\varepsilon>0$ there is $b(\varepsilon) \geq 0$ such that for any $g, h \in \Gamma$ possessing the axes and having the same translation length either their axes are asymptotic (=fellow travel each other) or the following inequality holds: $\ell\left(\sigma_{g}, \sigma_{h}, \varepsilon\right)<b(\varepsilon) a$, where $\sigma_{g}, \sigma_{h}$ are the axes of $g, h$ respectively, and $a$ is the amplitude of translation of $g, h$ on the axes. (See 2.2 for definition of $\ell$ ).

Lemma 6.2 (Separation of axes) If the group $\Gamma$ acts as a geometrically finite group of isometries of a pinched Hadamard manifold $X$, then $\Gamma$ satisfies the SA-property.

Proof. For $r>0$ denote by $b_{r}$ the maximum of cardinalities of the sets $S$ of elements of $\Gamma$, such that some orbit $S x, x \in X_{\Gamma}$ is contained in the ball of radius $r$. Since the action of $\Gamma$ on $X_{\Gamma}$ is cocompact, $b_{r}$ is finite. We assert that the $S A$-property is satisfied with $b(\varepsilon)=b_{\varepsilon}+1$.

Suppose the contrary then there is $\varepsilon>0$ and hyperbolic isometries $g, h \in$ $\Gamma$ such that $\ell\left(\sigma_{g}, \sigma_{h}, \varepsilon\right)>\left(b_{\varepsilon}+1\right) a$, where $\sigma_{g}, \sigma_{h}$ are axes of $g, h$ and $a$ is an amplitude of translation of $g, h$. Denote by $\sigma_{g}^{\prime}, \sigma_{h}^{\prime}$ the subsegments of $\sigma_{g}, \sigma_{h}$ respectively such that $\left|\sigma_{g}^{\prime}(t)-\sigma_{h}^{\prime}(t)\right| \leq \varepsilon, 0 \leq t \leq T$, and $T \geq\left(b_{\varepsilon}+1\right) a$. Note that $\sigma_{g}$ cannot stay within any horoball $B \in \mathcal{B}$, for time longer than $a$ since otherwise $g$ would preserve $B$ and hence would be parabolic. Hence, cutting $T$ by amount $a$ we may assume that $\left|\sigma_{g}^{\prime}(t)-\sigma_{h}^{\prime}(t)\right| \leq \varepsilon, 0 \leq t \leq T$, $T \geq b_{\varepsilon} a$ and $\sigma_{h}^{\prime}(0) \in X_{\Gamma}$. Changing if necessarily $g, h$ by their inverses, we may assume that the action of $g, h$ is coherent with the natural orientations of $\sigma_{g}^{\prime}, \sigma_{h}^{\prime}$. Then $g^{i} \sigma_{g}^{\prime}(0)=\sigma_{g}^{\prime}(i a) \in \sigma_{g}^{\prime}, h^{i} \sigma_{h}^{\prime}(0)=\sigma_{h}^{\prime}(i a) \in \sigma_{h}^{\prime}, i=0,1, \ldots, b_{\varepsilon}$ and we conclude that $\left|g^{i} \sigma_{g}^{\prime}(0)-h^{i} \sigma_{h}^{\prime}(0)\right| \leq \varepsilon, i=0,1, \ldots, b_{\varepsilon}$, hence $\mid \sigma_{g}^{\prime}(0)-$ $g^{-i} h^{i} \sigma_{h}^{\prime}(0) \mid \leq \varepsilon, i=0,1, \ldots, b_{\varepsilon}$. Thus we have $b_{\varepsilon}+1$ elements $g^{-i} h^{i} \sigma_{h}^{\prime}(0), i=$ $0,1, \ldots, b_{\varepsilon}$, inside the ball of radius $\varepsilon$ about $\sigma_{h}^{\prime}(0)$. All of these elements lie 
in $X_{\Gamma}$, since by our construction $\sigma_{h}^{\prime}(0) \in X_{\Gamma}$ ! By definition of $b_{\varepsilon}$, at least two of these elements coincide, say $g^{-i} h^{i} \sigma_{h}^{\prime}(0)=g^{-j} h^{j} \sigma_{h}^{\prime}(0), 0 \leq i \neq j \leq b_{\varepsilon}$. We conclude that $g^{i-j}=h^{i-j}$ for some $1 \leq i \neq j \leq b_{\varepsilon}$. It follows that $\left|\sigma_{g}(n(i-j))-\sigma_{h}(n(i-j))\right| \leq \varepsilon$ for all integral $n$ and hence the axes fellow travel each other. Hence they coincide by uniqueness of geodesics.

\section{Translation discreteness}

It is known that if $\Gamma$ is a group of isometries acting properly and cocompactly on a convex (in particular $\mathrm{CAT}(0)$ ) metric space $M$ then $\Gamma$ is translation discrete in the sense that translation numbers of its nontorsion isometries are bounded away from zero, [Con00], Corollary 3.8.

We generalize this to geometrically finite groups

Theorem 7.1 Suppose that the group $\Gamma$ acts as a nonelementary geometrically finite group of isometries of a pinched Hadamard manifold $X$. Then the action is translation discrete in a sense that the translation numbers of its hyperbolic isometries are bounded away from zero.

Proof. Let $\mathcal{B}$ be the horoball system attached to $\Gamma$ acting on $X$ and $X_{\Gamma}$ - the corresponding neutered space. Decreasing horoballs from $\mathcal{B}$ we may assume that that the distances between two distinct horoballs are bounded away from zero. Otherwise, there is a sequence of hyperbolic elements $g_{i}$ with translation numbers tending to 0 . Consider first the case when all the axes $A_{i}$ avoid $\mathcal{B}$. Fix any $x_{i} \in A_{i} \subset X_{\Gamma}$, then $\left\|g_{i} x_{i}-x_{i}\right\| \rightarrow 0$ with $i \rightarrow \infty$. Since $\Gamma \backslash X_{\Gamma}$ is compact there is a compact subset $D \subset X_{\Gamma}$ such that $\Gamma D=X_{\Gamma}$. For every $i$ there is $h_{i} \in \Gamma$, so that $h_{i} x_{i} \in D$. Choosing subsequence we may assume that $h_{i} x_{i} \rightarrow x \in D$. Then we have $\left|h_{i} g_{i} h_{i}^{-1} x-x\right| \leq \mid h_{i} g_{i} h_{i}^{-1} x-$ $h_{i} g_{i} h_{i}^{-1} h_{i} x|+| h_{i} g_{i} h_{i}^{-1} h_{i} x-h_{i} x|+| h_{i} x-x|=2| h_{i} x-x|+| h_{i} g_{i} x-h_{i} x \mid=$ $2\left|h_{i} x-x\right|+\left|g_{i} x-x\right| \rightarrow 0, i \rightarrow \infty$. This contradicts to the properness of $\Gamma$.

Now consider the case when there are infinitely many axes $A_{i}$ meeting $\mathcal{B}$ - we may then assume that all of them meet $\mathcal{B}$. Since each $g_{i}$ translates

nontrivially each horoball it visits, the translation length of $g_{i}$ is at least as large as the distance between distinct horoballs; thus it is bounded away from zero - a contradiction. 


\section{Free subgroups}

Koubi's criterion for free subgroups is as follows. We generalize it to our situation.

Lemma 8.1 (Freeness criterion)([Kou98], Lemma 2.4) Let g, $h$ be the isometries of a $\delta$-hyperbolic space $X$. Suppose that for a base point $x_{0}$ the following holds:

$$
\begin{gathered}
\left|g^{ \pm 1} x_{0}-h^{ \pm 1} x_{0}\right|>\max \left(\left|g x_{0}-x_{0}\right|,\left|h x_{0}-x_{0}\right|\right)+2 \delta \\
\left|g^{2} x_{0}-x_{0}\right|>\left|g x_{0}-x_{0}\right|+2 \delta \\
\left|h^{2} x_{0}-x_{0}\right|>\left|h x_{0}-x_{0}\right|+2 \delta
\end{gathered}
$$

Then $g, h$ freely generate the free group $F_{2}$.

Lemma 8.2 Let $X$ be a $\delta$-hyperbolic space and let $\Gamma \leq$ Isom $X$ be a nonelementary group acting properly on $X$. Suppose that the action $\Gamma$ on $X$ is translation discrete in the sense of Theorem 7.1 and satisfies the Separation Axes Property. Then there is a constant $m=m(\Gamma, X)$ such that for every hyperbolic $g_{0} \in \Gamma$ having axis and for any generating set $S$ there exists $s \in S$ such that $g=g_{0}^{m}$ and $h=s^{-1}$ s freely generate a free group of rank 2 .

Proof. Since $\Gamma$ on $X$ is translation discrete there is a constant $c_{0}>0$ such the translation length of any hyperbolic element, possessing an axis, is greater than $c_{0}$. Further, let $b_{0}=b(372 \delta)$ be the constant given by the SAproperty. We assert that $m>\frac{1}{c_{0}} 722 \delta+2 b_{0}$ is big enough to satisfy conclusion of the lemma. Suppose $g_{0} \in \Gamma$ has an axis $\sigma_{g}$ and let $S$ be a finite generating system for $\Gamma$. Since $\Gamma$ is nonelementary, there exists $s \in S$ not fixing the pair $\{\sigma(-\infty), \sigma(\infty)\}$. Then the axes $\sigma_{g_{0}}, \sigma_{h_{0}}$ of $g_{0}, h_{0}=s g_{0} s^{-1}$ have disjoint limit sets. By Separation Axes Property

$$
\ell_{0}=\ell\left(\sigma_{g_{0}}, \sigma_{h_{0}}, 380 \delta\right) \leq b_{0}\left\|g_{0}\right\| .
$$

By the choice of $m$ the translation length $m\left\|g_{0}\right\|$ of $g=g_{0}^{m}$ is greater than $722 \delta+2 b_{0}\left\|g_{0}\right\|$. Reparameterize the geodesics so that at the moment $t=0$ they are located at the points $x_{0} \in \sigma_{g}=\sigma_{g_{0}}, y_{0} \in \sigma_{h}=\sigma_{h_{0}}$, which are the closest ones among the points of these axes. 
We shall verify conditions of the criterion of freeness 8.1. We start with the condition

$$
\left|g x_{0}-h x_{0}\right|>\max \left\{\left|g x_{0}-x_{0}\right|,\left|h x_{0}-x_{0}\right|\right\}+2 \delta .
$$

Remote case: Suppose that $\left|x_{0}-y_{0}\right|=\left|h x_{0}-h y_{0}\right|>180 \delta$. The 5-gon $\left[g x_{0}, x_{0}, y_{0}, h y_{0}, h x_{0}\right]$ satisfies assumptions of Lemma 2.5. Indeed, the lengths of the sides $\left[g x_{0}, x_{0}\right],\left[h y_{0}, h x_{0}\right]$ are at least $180 \delta$ each. Angles at the points $x_{0}, y_{0}, h y_{0}$ are all obtuse, since, for example, $x_{0}$ is the nearest to $y_{0}$ point on $\sigma_{g}$. Hence by Lemma $2.4\left(g x_{0} \cdot y_{0}\right)_{x_{0}}<8 \delta \leq 14 \delta$.

We conclude that

$$
\begin{gathered}
\left|g x_{0}-h x_{0}\right|>\left|g x_{0}-x_{0}\right|+\left|h y_{0}-y_{0}\right|+2\left|x_{0}-y_{0}\right|-168 \delta \geq \\
\left|g x_{0}-x_{0}\right|+\left|h x_{0}-x_{0}\right|-168 \delta .
\end{gathered}
$$

Applying lemma 2.5 to the 4-gon $\left[x_{0}, y_{0}, h y_{0}, h x_{0}\right]$ we obtain

$$
\left|h x_{0}-x_{0}\right|>2\left|x_{0}-y_{0}\right|+\left|h y_{0}-y_{0}\right|-168 \delta>180 \delta,
$$

hence, combining with the above we obtain

$$
\left|g x_{0}-h x_{0}\right|>\max \left\{\left|g x_{0}-x_{0}\right|,\left|h x_{0}-x_{0}\right|\right\}+2 \delta .
$$

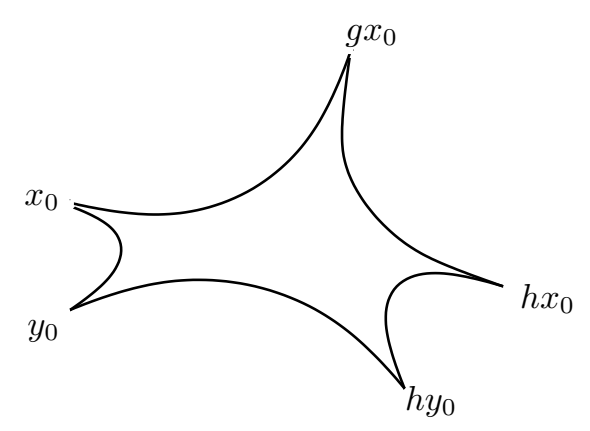

Figure 6: Geodesic 5-gon.

Nearby case: Suppose that $\left|x_{0}-y_{0}\right|=\left|h x_{0}-h y_{0}\right| \leq 180 \delta$. Then by lemma 2.6 applied to the 5 -gon $\left[g x_{0}, x_{0}, y_{0}, h y_{0}, h x_{0}\right]$ we have

$$
\left|g x_{0}-h x_{0}\right|>\left|g x_{0}-x_{0}\right|+\left|h y_{0}-y_{0}\right|-360 \delta-2 b_{0}|| g_{0}|| \geq
$$


(since both summands below are $\geq 722 \delta+2 b_{0}\left\|g_{0}\right\|$ )

$$
\max \left\{\left|g x_{0}-x_{0}\right|,\left|h y_{0}-y_{0}\right|\right\}+362 \delta \geq
$$

(since $\left.\left|h y_{0}-y_{0}\right| \geq\left|h x_{0}-x_{0}\right|-360 \delta\right)$ )

$$
\max \left\{\left|g x_{0}-x_{0}\right|,\left|h x_{0}-x_{0}\right|\right\}+2 \delta .
$$

It remains to verify the conditions $\left|g^{2} x_{0}-x_{0}\right|>\left|g x_{0}-x_{0}\right|+2 \delta,\left|h^{2} x_{0}-x_{0}\right|>$ $\left|h x_{0}-x_{0}\right|+2 \delta$.

The first is clear since $\left|g^{2} x_{0}-x_{0}\right|=2\left|g x_{0}-x_{0}\right|$. For the second, since $x_{0}$ does not lie on the axis of $h$, we shall consider the remote and nearby cases. If $\left|x_{0}-y_{0}\right|=\left|h x_{0}-h y_{0}\right|>180 \delta$ then applying Lemma 2.5 to the 4-gon $\left[x_{0}, y_{0}, h^{2} y_{0}, h^{2} x_{0}\right]$ we obtain

$$
\begin{gathered}
\left|h^{2} x_{0}-x_{0}\right|>2\left|x_{0}-y_{0}\right|+\left|h^{2} y_{0}-y_{0}\right|-168 \delta=2\left|x_{0}-y_{0}\right|+2\left|h y_{0}-y_{0}\right|-168 \delta \\
>360 \delta+\left|h y_{0}-y_{0}\right|-168 \delta>\left|h y_{0}-y_{0}\right|+2 \delta .
\end{gathered}
$$

In the nearby case we have $\left|x_{0}-y_{0}\right|=\left|h x_{0}-h y_{0}\right| \leq 180 \delta$ and then

$$
\left|h^{2} x_{0}-x_{0}\right| \geq\left|h^{2} y_{0}-y_{0}\right|-2\left|x_{0}-y_{0}\right| \geq 2\left|h y_{0}-y_{0}\right|-360 \delta>\left|h y_{0}-y_{0}\right|+360 \delta \text {. }
$$

Proof of the theorem 1.1. Suppose that $\Gamma$ is a nonelementary geometrically finite group of isometries of a pinched Hadamard manifold $X$. Denote by $X_{\Gamma}$ the corresponding neutered space. Since $\Gamma$ acting on $X_{\Gamma}$ is proper and cocompact it satisfies Separation Axes Property. By Lemma 6.2 $\Gamma$ acting on $X$ satisfies Separation Axes Property too. Moreover by Theorem 5.2, $\Gamma$ uniformly contains hyperbolic elements. Hence by lemma $8.2 \Gamma$ uniformly contains a free group of rank two. 


\section{References}

$\left[\mathrm{ABC}^{+} 91\right]$ J.M. Alonso, T. Brady, D. Cooper, V. Ferlini, M. Lustig, M. Mihalik, M. Shapiro, and H. Short. Notes on word hyperbolic groups. In Group theory from a geometrical viewpoint (Trieste, 1990), pages 3-63. World Sci. Publishing, River Edge, NJ, 1991. Edited by H. Short.

[AN02] Roger C. Alperin and Guennadi A. Noskov. Uniform growth, actions on trees and $\mathrm{GL}_{2}$. In Computational and statistical group theory (Las Vegas, NV/Hoboken, NJ, 2001), volume 298 of Contemp. Math., pages 1-5. Amer. Math. Soc., Providence, RI, 2002.

[Ave72] André Avez. Entropie des groupes de type fini. C. R. Acad. Sci. Paris Sér. A-B, 275:A1363-A1366, 1972.

[BGS85] Werner Ballmann, Mikhael Gromov, and Viktor Schroeder. Manifolds of nonpositive curvature. Birkhäuser Boston Inc., Boston, MA, 1985.

[Bow95] B.H. Bowditch. Geometrical finiteness with variable negative curvature. Duke Math. J., 77(1):229-274, 1995.

[CDP90] M. Coornaert, T. Delzant, and A. Papadopoulos. Géométrie et théorie des groupes. Springer-Verlag, Berlin, 1990. Les groupes hyperboliques de Gromov. [Gromov hyperbolic groups], With an English summary.

[Con00] Gregory R. Conner. Translation numbers of groups acting on quasiconvex spaces. In Computational and geometric aspects of modern algebra (Edinburgh, 1998), pages 28-38. Cambridge Univ. Press, Cambridge, 2000.

[Del96] Thomas Delzant. Sous-groupes distingués et quotients des groupes hyperboliques. Duke Math. J., 83(3):661-682, 1996.

[dlH02] Pierre de la Harpe. Uniform growth in groups of exponential growth. In Proceedings of the Conference on Geometric and Combinatorial Group Theory, Part II (Haifa, 2000), volume 95, pages 1-17, 2002. 
[EMO01] A. Eskin, S. Mozes, and H. Oh. Uniform exponential growth for linear groups. Preprint, University of Chicago , http://zaphod.uchicago.edu/ eskin/, 2001.

[Far95] Benson Farb. Combing lattices in semisimple Lie groups. In Groups-Korea '94 (Pusan), pages 57-67. de Gruyter, Berlin, 1995.

[GdlH97] R. Grigorchuk and P. de la Harpe. On problems related to growth, entropy and spectrum in group theory. J. of Dynamical and Control System, 3(1):51-89, 1997.

[GG00] F. Grunewald and G.Noskov. Largeness of certain hyperbolic lattices. Preprint, Duesseldorf University, 2000.

[Gro87] M. Gromov. Hyperbolic groups. In Essays in group theory, volume 8 of Math. Sci. Res. Inst. Publ., pages 75-263. Springer, New York, 1987.

[Gro99] Misha Gromov. Metric structures for Riemannian and nonRiemannian spaces. Birkhäuser Boston Inc., Boston, MA, 1999. Based on the 1981 French original [MR 85e:53051], With appendices by M. Katz, P. Pansu and S. Semmes, Translated from the French by Sean Michael Bates.

[HIH77] Ernst Heintze and Hans-Christoph Im Hof. Geometry of horospheres. J. Differential Geom., 12(4):481-491 (1978), 1977.

[Kou98] Malik Koubi. Croissance uniforme dans les groupes hyperboliques. Ann. Inst. Fourier (Grenoble), 48(5):1441-1453, 1998.

[Man81] Anthony Manning. More topological entropy for geodesic flows. In Dynamical systems and turbulence, Warwick 1980 (Coventry, 1979/1980), pages 243-249. Springer, Berlin, 1981.

[Ol'91] A. Yu. Ol'shanskiu. Periodic quotient groups of hyperbolic groups. Mat. Sb., 182(4):543-567, 1991.

[Wil02] John S. Wilson. On exponential growth and uniformly exponential growth for groups. . Preprint, University of Birmingham, 2002. 
Roger C. Alperin:

Dept. of Mathematics,

San Jose State University

San Jose, CA 95192

E-mail: alperin@math.sjsu.edu

Gennady A. Noskov:

Institute of Mathematics,

Russian Academy of Sciences,

Pevtsova 13, Omsk, 644099, Russia

and

Fakultät für Mathematik

Universität Bielefeld

Postfach 100131,

D-33501 Bielefeld,

Germany

E-mail:noskov@mathematik.uni-bielfeld.de 